\title{
Technical improvements to the projection tachistoscope
}

\author{
WILHELM R. GLASER \\ University of Tübingen, Tübingen, Federal Republic of Germany
}

\begin{abstract}
Three disadvantages of the usual projection tachistoscope are discussed. Because of low mechanical precision, stimulus alignment is difficult. Because of rather low focal distances of the projection lenses, the stimuli provide visual angles that are often larger than desired, and the photographic shutters are noisy, are relatively inert, and have a limited service life. A new construction is described that avoids these disadvantages with a modified configuration of commercial elements: stable and precise mounting of the single channels, a double-lens projection system, and a noiseless, fast mechanical shutter based on a limited-rotation motor.
\end{abstract}

Since the microcomputer has become standard equipment in the psychological laboratory, there has been an increasing trend to use its CRT monitor as a tachistoscope. A great deal of research with controlled visual stimulation can be conducted at drastically lowered technical costs on computers, because the stimuli are designed, changed, and displayed via software control simply by using adequately written programs.

However, because the CRT-generated picture is sequentially written by a single rapidly deflected electron beam, conventional tachistoscopes need to be used for some applications. They are particularly useful if structurally rich stimuli that cover large areas are to be presented at intervals and durations of less than the 16.7 or $20 \mathrm{msec}$ (depending on line frequency, $60 \mathrm{or} 50 \mathrm{~Hz}$ ) of the electronic raster pulse or beyond an integral multiple thereof. The traditional tachistoscopes provide timing that is unrestricted by the video time base, a truly synchronous exposure of the whole stimulus field, a practically unlimited spatial resolution, and, when using mechanical shutters, a complete and rapid fade-out after exposure (see Rayner \& Pollatsek, 1983).

The mirror and the projection tachistoscopes differ in the following ways:

1. The mirror tachistoscope requires that the stimuli be prepared in the to-be-presented size, whereas the projection tachistoscope requires $35-\mathrm{mm}$ transparencies. Therefore, the stimulus materials are inconvenient for the mirror tachistoscope. On the other hand, they can be made by every graphic technique, whereas the stimuli for the projection tachistoscope must be photographed.

The development of the instrument described here was financially supported by Grant Gl 121/1 of the Deutsche Forschungsgemeinschaft. I am indebted to Hugo Kehrer and Gerhard Schnerr, the chiefs of the mechanical and electronics workshop of the Psychological Institute at Tübingen. They designed and manufactured the mechanical mounting of the instrument and the electronic driver for the shutter. The help of Sally Bellows in answering questions concerning English usage is gratefully acknowledged. Requests for reprints should be sent to Wilhelm R. Glaser, Psychological Institute, University of Tübingen, Friedrichstrasse 21, D-7400 Tübingen, Federal Republic of Germany.
2. In addition to the size of the stimuli, the viewing distance and the viewing angle of the stimuli are fixed by the construction of the mirror tachistoscope. The projection tachistoscope, particularly with the modifications presented below, permits wide and independent variations of stimulus size, viewing distance, and viewing angle. Because in the mirror tachistoscope the stimuli are large in relation to the mechanical tolerances of the apparatus, the alignment of stimuli in different channels is superior to that of the usual projection tachistoscope. Furthermore, if high acuity and resolution are required, then the direct view of the stimuli in the mirror tachistoscope that has no picture-generating optics (except the plane-parallel semipermeable glass plates) is best.

3 . With the projection tachistoscope it is easy to provide computer-controlled automatic stimulus selection because fast and reliable random-access slide projectors are commercially available. Devices that automatically change the stimulus cards in a mirror tachistoscope are expensive, inconvenient, and slow; thus automatic control is often rejected and the stimuli changed by hand.

4. In the mirror tachistoscope, the stimuli are illuminated by electronically controlled gas discharge lamps. They are practically not inert at stimulus onset and reach their full intensity within less than $1 \mathrm{msec}$. The stimulus offset, however, shows an exponential decay with time constants in the range of some $15 \mathrm{msec}$. The color and intensity of light emission change rapidly when these lamps grow old, so that repeated adjustments become necessary. In the projection tachistoscope, the projection beams are generated by incandescent lamps and controlled by photographic shutters. These shutters exhibit some disadvantages that are discussed below in more detail.

As a whole, the standard commercial projection tachistoscope, as described in most catalogs, has three deficiencies when compared with the mirror tachistoscope. These can be overcome by the improvements described below.

First, it is usually difficult to adjust the optical axes of two or more channels with the necessary precision for the 
spatial alignment of the stimulus components (see Fraser, Lishman, \& Parker, 1987). This problem is solved by putting each channel on a separate optical bench with stable mounting and exact adjustment screws (Figure 2).

Second, the usual projection lenses have a fixed focal distance of about $90 \mathrm{~mm}$ so that the $24 \times 36 \mathrm{~mm}$ slide is projected at $53 \times 80 \mathrm{~cm}$ from a distance of $2 \mathrm{~m}$. A subject sitting $1.5 \mathrm{~m}$ away from this projection has a viewing angle of about $20^{\circ} \times 30^{\circ}$, which is too large for most psychological applications. The use of lenses with larger focal distances is restricted because their correspondingly larger apertures require photographic shutters with large diameters. Such shutters are heavy, expensive, noisy, and relatively slow. They require strong power electronics for operation and have a limited service life. Therefore, an optical system is required with a long and variable effective focal distance of usually more than $180 \mathrm{~mm}$. It should have a small aperture at the shutter location. This problem is solved by using two lenses, as shown in Figure 1a. The first lens $(1: 2.8 / 50 \mathrm{~mm})$ generates a reduced, real, and inverted image, which is projected onto the screen by the second lens $(1: 2.5 / 90 \mathrm{~mm})$. The resulting effective focal distance of this system is given by the reduction ratio of the first lens $X$ the focal distance of the second. This reduction ratio depends only on the distance between the film inside the projector and the first lens. It is therefore easily adjusted within a broad range by displacing the first lens on the optical bench. The distance between both lenses is equal to the sum of the image distance from the first lens and the focal distance of the second lens for a clear projection on the screen.

Third, the photographic shutters have some disadvantages if used in projection tachistoscopes. As mentioned, they are unfavorable with large lenses, and they produce a loud noise during operation that is an acoustical nuisance. Their inertia cannot be ignored; for example, a frequently used shutter in Europe (Compur electronicm 3 with 40-mm aperture) has a latency for opening or closing of about $8 \mathrm{msec}$ and a transition time of about $6 \mathrm{msec}$. The minimum full-open time is about $10 \mathrm{msec}$, and the minimum interval between two cycles is $500 \mathrm{msec}$. Using the double-lens optics described above also solves this problem.

The photographic shutter is replaced by a simple flap that is fixed by a ring and a binding screw on the axis of a limited-rotation motor (R4-155; MFE Corporation,

Projector

with Mirror, Lamp, Thermal Filter,

First Lens Shutter

Second Lens

Screen

and Condenser Lenses

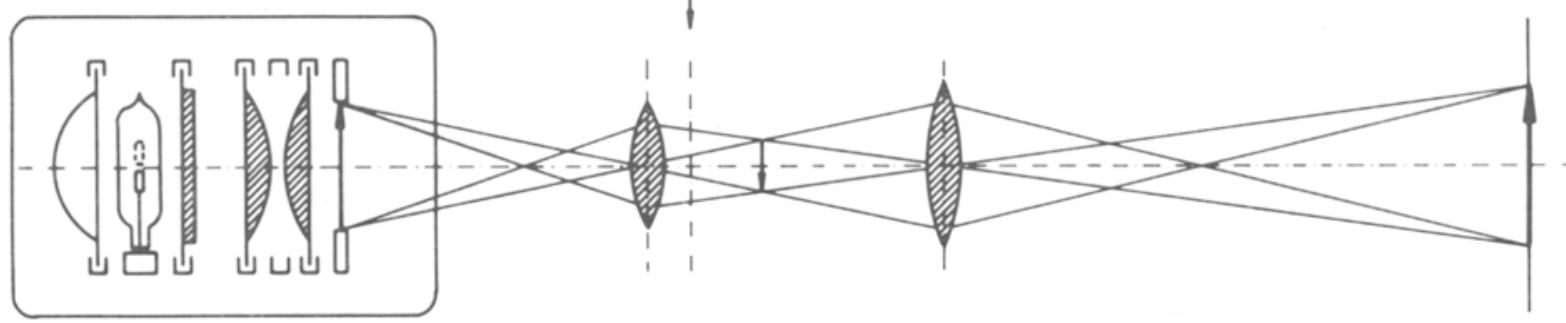

(a)

Slot No. 12234 Slide
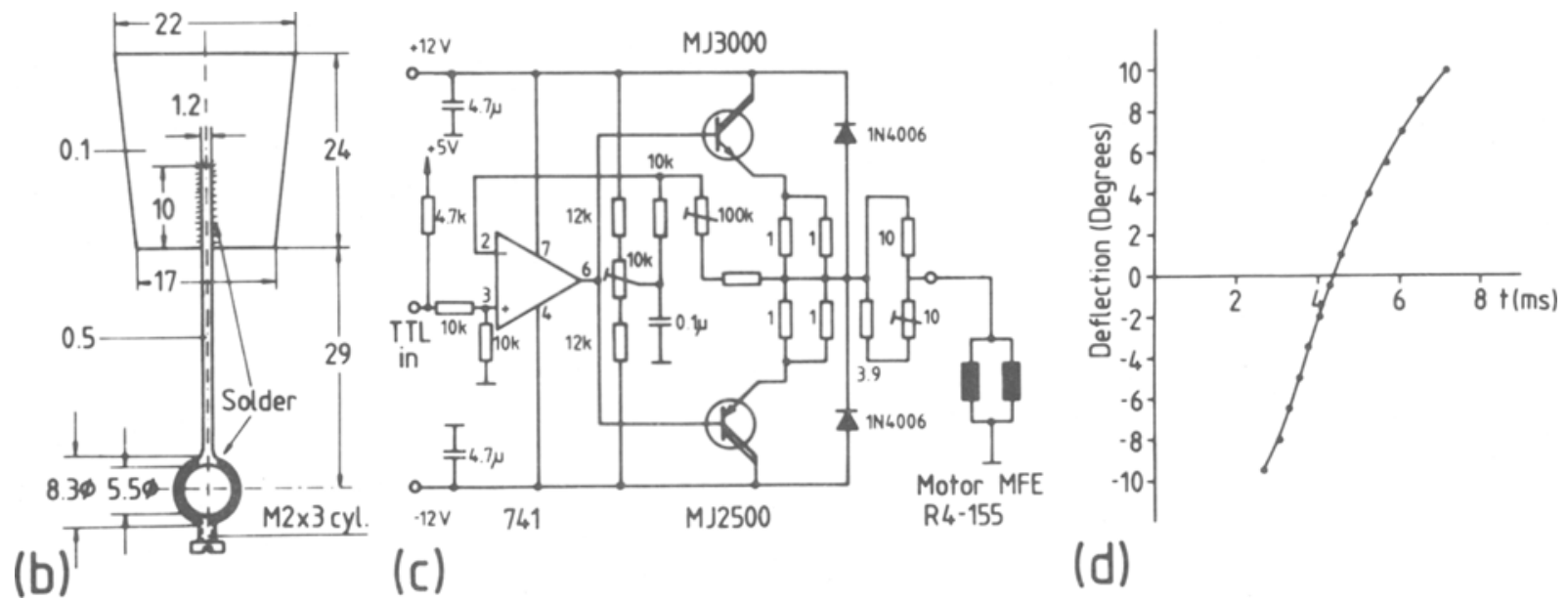

(c)

(d)

Figure 1. Essentials of the improved tachistoscope: (a) the modified optical system (not drawn to correct scale), (b) the flap, (c) the electronic driver, (d) the time diagram of the flap's response to a step function at driver input at $t=0$. The flap is made of spring-quality brass, 0.1 and $0.5 \mathrm{~mm}$ thick, with tin soldering. All measures are given in millimeters. 
Salem, NH 03079). This motor is specially designed for optical scanning and related purposes. The form and dimensions of the flap are shown in Figure lb. It is made from spring-quality brass of $0.1-\mathrm{mm}$ thickness for the trapezoid and of $0.5-\mathrm{mm}$ thickness for the stem. The axis of the motor is mounted in parallel to the optical axis of the projection system (see Figure 2, No. 3) so that the trapezoidal part of the flap interrupts or releases the projection beam between first lens and reduced image if the motor turns by $\pm 11^{\circ}$.

Of course, the usefulness of this simple shutter mechanism depends on the flap's reaching a sufficient velocity. The flap has a weight of $1.3 \mathrm{~g}$ and an inertia of $7.3 \mathrm{~g} \times \mathrm{cm}^{2}$. Together with the physical characteristics of the motor (see MFE Corporation, 1980), this leads to a calculated natural frequency of $70 \mathrm{~Hz}$ and thus complete peak-to-peak movement in $7 \mathrm{msec}$. The driving electronics must provide a fast current change against coil inductance and back electromotive force. The circuitry, as shown in Figure 1c, is adequate for that. We choose a steady-state voltage of $\pm 3.5 \mathrm{~V}$, thus obtaining a steady deflection of the flap by $\pm 11^{\circ}$ with a current of $\pm 320 \mathrm{~mA}$. To judge the usefulness of this shutter, the time-way diagram of the flap corresponding to the step function of the electronic driver at $t=0$ was measured. It is shown in Figure 1d. Apparently, there is a latency of $2.5 \mathrm{msec}$. By $0.5 \mathrm{msec}$ later, a distance of only $3^{\circ}$ is covered, whereas the speed has almost completely reached its maximum of $5.7^{\circ}$ per millisecond. After an additional $3 \mathrm{msec}, 18^{\circ}$ are covered, followed by a decelerated transition into the final position during the next $1.5 \mathrm{msec}$. All of these values proved highly stable and reproducible within $\pm 10-\mu \mathrm{sec}$ limits. Therefore, the tachistoscope has a latency of $3 \mathrm{msec}$ and a transition time of $3 \mathrm{msec}$ when the vertical measure of the projection beam is equal to the central $15^{\circ}$ of the flap's movement. With the dimensions of the flap given in Figure $1 \mathrm{~b}$, a beam of about $13 \mathrm{~mm}$ diameter can be switched. Because the system of projection lenses described above provides a beam diameter in this magnitude at the shutter location, a physically precise tachistoscope results.

Compared with the conventional projection tachistoscope, the system described here has the following advantages: (1) The channels can be precisely adjusted for an exact superposition of stimulus components. (2) The system of two projection lenses provides stimulus dimensions that are more adequate for most psychological purposes. These dimensions can easily be varied and adjusted to the desired projection and viewing distances. The possible use of the full $24 \times 36 \mathrm{~mm}$ film field for small visual angles increases the precision of the stimulus alignment between the channels. (3) The mechanical shutter is practically noiseless and about twice as fast as the photographic shutter. It permits the fast repetition of the cycle up to frequencies of some $70 \mathrm{~Hz}$, that is, sufficiently beyond fusion frequency. Because it has only one moving part, its service life is almost unlimited.
There are also disadvantages, however. The mechanical mounting based on one optical bench per channel is not inexpensive, although it poses no problems for a mechanical workshop. The limited-rotation motor is more expensive than the cheaper photographic shutters. Also, because there are two lenses in each channel, the intensity of the projection is lowered by about the factor two. These lenses must be carefully selected to maintain a high resolution because the aberrations are superposed on one another. After extensive tests and calculations, we chose an enlargement lens (Rodenstock Rodagon 1:2.8/50 mm) for the first lens and a projection lens (Leitz Colorplan $1: 2.5 / 90 \mathrm{~mm}$ ) for the second lens. Excellent results were obtained with two high-fidelity lenses (Rodenstock Apo-Rodagon 1:2.8/50 mm; Kodak Retinar 2000 $1: 2.5 / 93 \mathrm{~mm}$ ); however, they were very expensive (about $\$ 380$ for each channel).

Building the tachistoscope is not particularly difficult for the mechanical and electronics workshops usually found at experimental psychology laboratories because all critical parts-that is, random-access projectors, lenses, optical benches, and electronic components-are commercially available. Only the flap must be assembled according to specific directions (see Figure $1 b$ ). ${ }^{1}$ One minor technical problem must be mentioned: According to the Maxwell theory of projection systems, the condenser lenses of the projector must be adjusted to the position of the first projection lens in order to provide constant illumination of the whole stimulus field. We obtained the best results with Kodak Carousel projectors by arranging the thermal filter and two plane-convex condenser lenses as shown in Figure 1a.

The essential mechanical details of a single channel of the instrument are shown in Figure 2. Two channels can easily be mounted side by side. A third and a fourth channel are best located above the first and second with some lateral displacement. Two minor details that are not contained in Figure 2 need to be mentioned. Because the usual lens is removed from the projector, some spread light emerges. It is kept away from the screen by a circular shield of black cardboard with about $15 \mathrm{~cm}$ diameter around the first lens (marked number 2 in Figure 2). Furthermore, we mounted a plane-parallel glass plate of $6 \times 6 \mathrm{~cm}$ with an angle of $45^{\circ}$ into the projection beam beyond the second lens (number 4 in Figure 2) in order to mirror some light on a phototransistor. This phototransistor allows computer monitoring of the real stimulus onset. We use its signal for an exact reaction time measurement and a current check of correct shutter operation.

The instrument has been used in about 20 experiments since 1985 with about 200,000 double-stimulation trials. The only technical problem was that the stem of the flap showed a tendency to break near the soldering point with the mounting ring after some 20,000 trials, due to the high torsional acceleration. This problem apparently can be solved by broadening the connection with the ring and making round the adjacent corners, as is shown in 


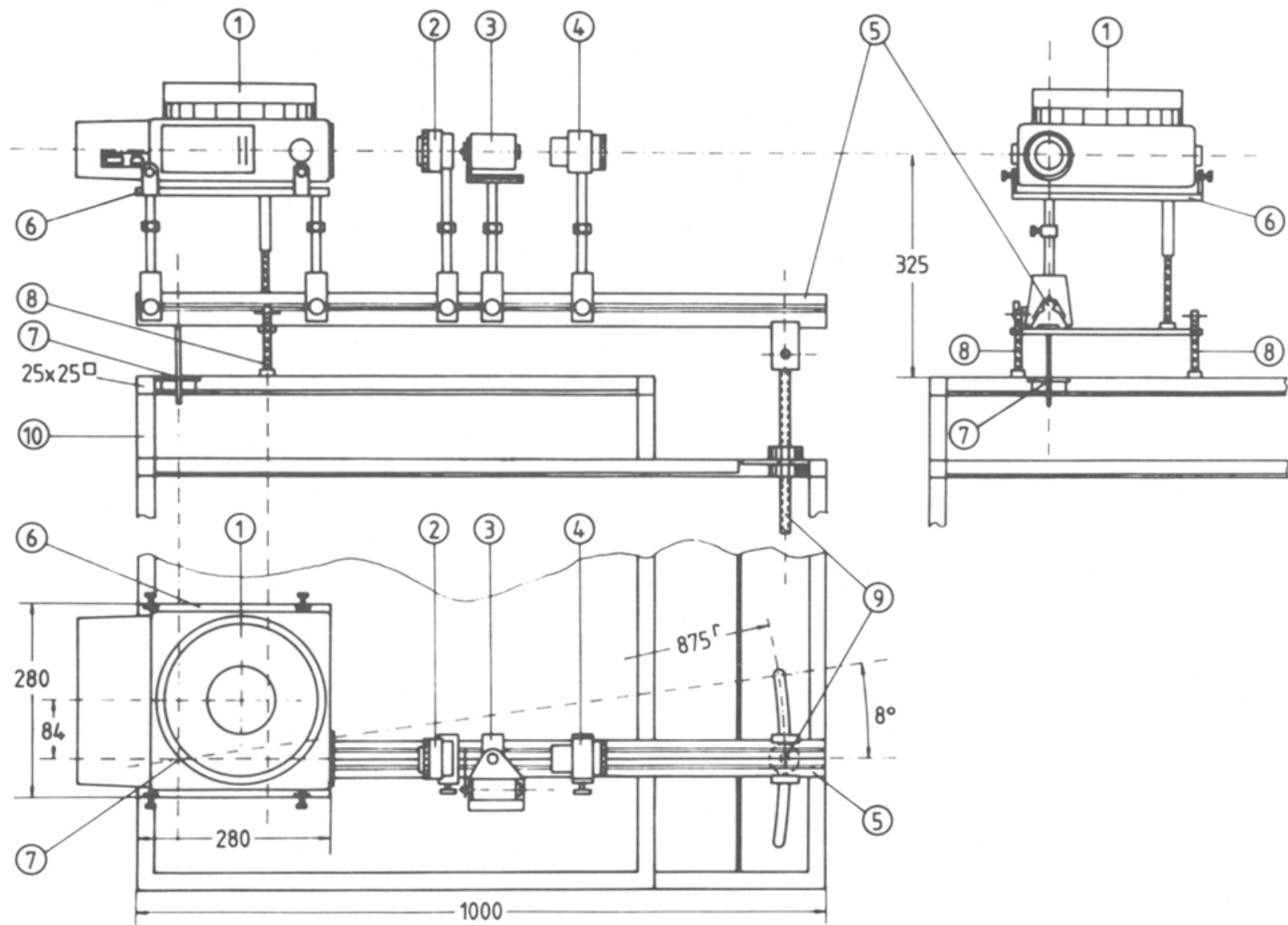

Figure 2. Mechanical mounting of one channel: (1) projector, (2) first lens (without spread-light shield), (3) torque motor with flap, (4) second lens, (5) optical bench, (6) projector support with four adjustment screws, (7) pivot for vertical axis (no load), (8) two supporting bolts (lateral axis; adjustment around longitudinal axis), (9) third supporting bolt (with bearing nut for vertical adjustment, circular slot for lateral adjustment, and locking counter-nut), (10) frame (welded section iron; wooden panels). Measures are given in millimeters.

Figure 1b. We achieved the complete timing of stimulus selection and exposure using a Hewlett-Packard Type 1000 minicomputer, which permitted efficient timing, control, and registration of all experimental variables with low programming effort.

\section{REFERENCES}

Fraser, I. H., Lishman, J. R., \& Parker, D. M. (1987). Temporal manipulation of stimulus patterns using the Apple II: Tachistoscopic and part presentation. Behavior Research Methods, Instruments, \& Computers, 19, 315-318.
MFE CoRporation. (1980). Limited rotation motors (Data sheet 20M/2/80). Salem, NH: Author.

Rayner, K., \& Pollatsek, A. (1983). Is visual information integrated across saccades? Perception \& Psychophysics, 34, 39-48.

\section{NOTE}

1. A small number of flaps can be obtained without charge from the author.

(Manuscript received December 2, 1987; revision accepted for publication May 13, 1988.) 